\title{
Atomistic dynamics of deformation, fracture, and joining of individual single-walled carbon nanotubes
}

\author{
Koji Asaka \\ Special Research Project on Nanoscience, Graduate School of Pure and Applied Sciences, \\ University of Tsukuba, Tsukuba 305-8571, Japan \\ Tokushi Kizuka \\ Institute of Materials Science, Graduate School of Pure and Applied Sciences, \\ University of Tsukuba, and PREST, JST, Tsukuba 305-8573, Japan
}

(Received 12 April 2005; published 26 September 2005)

\begin{abstract}
The tensile deformation process of individual single-walled carbon nanotubes (SWCNTs) was in situ observed with measurements of the mechanical properties by transmission electron microscopy combined with the functions of scanning probe microscopy. The values of Young's modulus, tensile stress and strain at the fracture of SWCNTs are $950 \pm 130 \mathrm{GPa}, 25 \pm 1 \mathrm{GPa}$, and $0.30 \pm 0.03$, respectively. It was shown that two tips of fractured SWCNTs can be recovered to produce a single tubular structure by contact under compressive force and bias voltage. The in situ observations also demonstrated the formation of carbon monatomic wires during the fracture process of SWCNTs.

DOI: 10.1103/PhysRevB.72.115431

PACS number(s): 81.07.De, 62.20.-x, 61.46.+w, 81.07.-b
\end{abstract}

\section{INTRODUCTION}

Since the discovery of carbon nanotubes, ${ }^{1}$ their unique mechanical and electrical properties have been studied to develop nanometer scale technology. ${ }^{2-8}$ In particular, theoretical analyses of mechanical properties have predicted that single-walled carbon nanotubes (SWCNTs) have a Young's modulus in excess of $1000 \mathrm{GPa}$ and a strain of up to $0.5 .^{9-17}$ In previous experiments, the modulus and strain widely ranged from 90 to $4000 \mathrm{GPa}$ and 0.01 to 0.1 , respectively. ${ }^{18-25}$ Such mechanical properties are associated with the atomistic structural dynamics during deformation. However, no experiment has been performed to analyze the relationship between mechanical properties and structural dynamics, because it was difficult to perform the manipulation of individual SWCNTs and to simultaneously observe tensile deformation at an atomic scale. We recently developed an experimental method to analyze the atomistic mechanics of nanometer-sized materials. ${ }^{26}$ In this report, we demonstrate in situ observations of atomistic structural dynamics of individual SWCNTs during tensile deformation and simultaneous measurements of the mechanics of materials. We also observed their joining process, which is a fundamental process in its application to nanometer scale devices.

\section{EXPERIMENTAL METHOD}

We prepared SWCNTs inside a transmission electron microscope combined with the functions of scanning probe microscopes. ${ }^{26}$ The cantilever coated with a thin gold film, for atomic force microscopy, was fixed on the front of a tube-type piezo. A thin amorphous carbon plate with a nanometer-sized tip was fixed on another specimen holder. The tip of the cantilever was then brought into contact with the opposite tip of the amorphous carbon plate at room tem- perature with an applied voltage of $1.6-2.2 \mathrm{~V}$ between the two tips, and subsequently retracted by driving the piezo. Afterwards, the surfaces of both tips were covered with graphitic layers, and upon subsequent contact, a SWCNT emerged between the two surfaces. This preparation method was different from previously reported methods of peeling off a graphitic layer ${ }^{27}$ and the electron beam irradiation on amorphous carbon films. ${ }^{28}$ The SWCNT was then retracted along the direction of a long-axis by driving the piezo at atomistic intervals. Structural variations during tensile deformation were directly observed by in situ high-resolution transmission electron microscopy. High-resolution imaging was performed at an accelerating voltage of $200 \mathrm{kV}$ with a TV-rate system. High-resolution images were recorded on videotapes with a time resolution of $16 \mathrm{~ms}$. The base pressure of the specimen chamber of the microscope was 1 $\times 10^{-5} \mathrm{~Pa}$. Variations in force, current, bias voltage, and the voltages applied to the piezo during deformation were simultaneously measured at intervals of $4 \mathrm{~ms}$. The length and width of the contacted regions during deformation were estimated from each video field image. In this study, we precisely observed the shape of the cantilevers by scanning electron microscopy and estimated the force constant based on calculations via finite element methods, because the commercial micrometer cantilevers ranged in size and the force constant depends on the length, width, and thickness. Since any kind of microscopy with micrometer resolution can be used to estimate the actual force constant of each cantilever, we selected scanning electron microscopy for the characterization of the cantilevers. If an average value of the force constant is applied for the estimation of the force, accidental errors will creep into the final values.

\section{RESULTS AND DISCUSSION}

Figures 1(a)-1(c) show a time-sequential series of highresolution images of the tensile deformation of a SWCNT. 

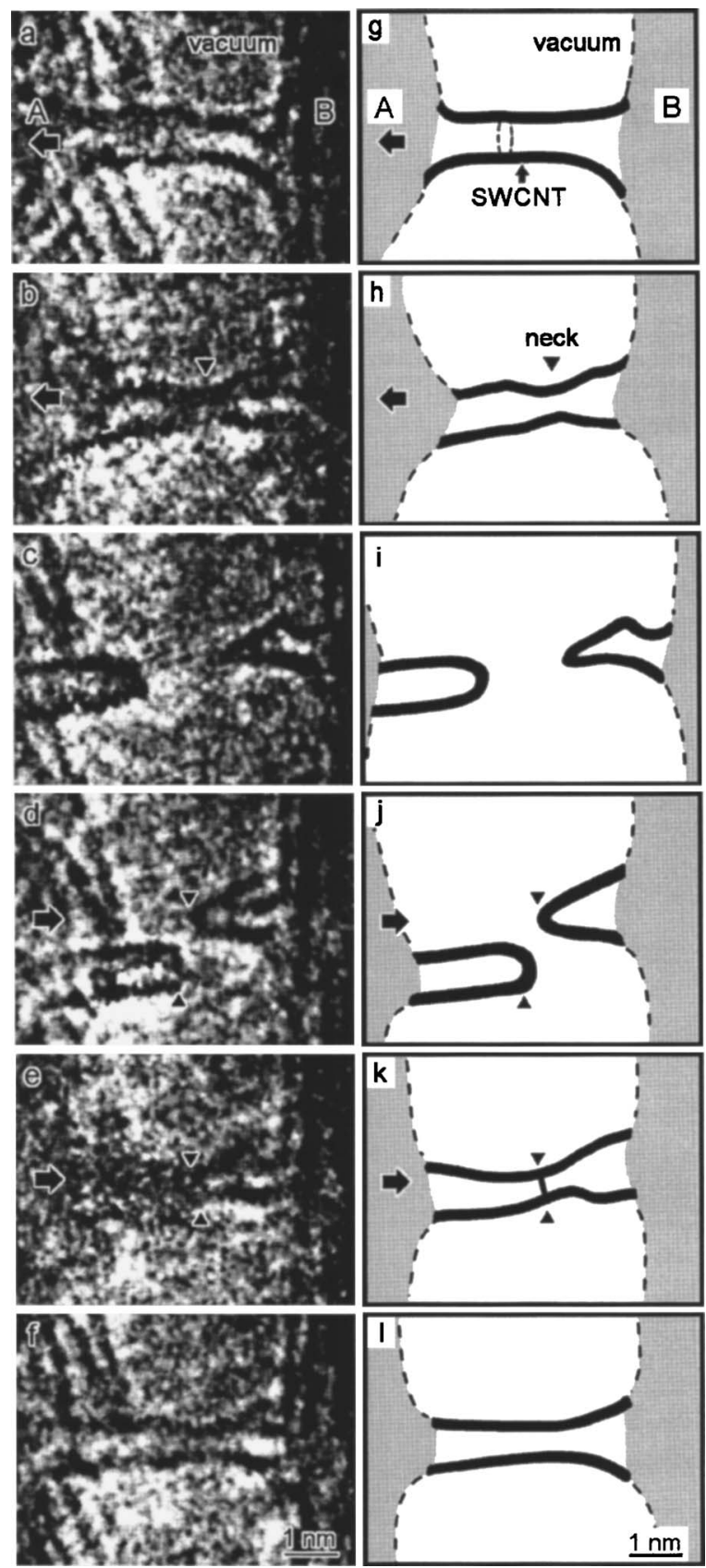

FIG. 1. Time-sequential series of high-resolution images of a single-walled carbon nanotube (SWCNT): (a)-(c) tensile deformation process, (d)-(f) joining process. The illustrations in (g)-(l) correspond to the images in (a)-(f). The SWCNTs formed between the graphitized surfaces of a tip of the cantilever (A) and that of the carbon plate (B). The minimum diameter of the SWCNT is $0.9 \mathrm{~nm}$ in (a). With stretching along the direction indicated by the bold arrow in (a)-(b), a neck forms as seen in (b), and the SWCNT finally fractures as in (c). After complete fracture, when the tip of the SWCNT on the right-hand side compresses that of the opposite SWCNT at the applied voltage of $2.2 \mathrm{~V}$, one stable tubular structure is reproduced as shown in (f).

Figures 1(g)-1(i) show illustrations corresponding to Figs. 1(a)-1(c). The left-hand and right-hand regions in Fig. 1 are graphitic surface layers of the tip of a cantilever (A) and that of the carbon plate (B). First, between the surface layers of the two tips, the SWCNT with a minimum diameter of $0.9 \mathrm{~nm}$ is observed [Fig. 1(a)]. This image is a typical highresolution image of a SWCNT, as reported by Iijima. ${ }^{29}$ The two edges of the SWCNT connect with the surface layers of tips A and B. As the SWCNT is stretched along the direction indicated by the bold arrows in Figs. 1(a) and 1(b), a neck forms at a part of the wall, as designated by the arrow head in Fig. 1(b). This indicates that buckling occurs in the SWCNT due to tensile deformation. Subsequently, the SWCNT breaks completely at the neck. Each tip of the broken SWCNT is closed, as shown in Fig. 1(c). The tip shape is similar to that of a carbon nano-horn with a cone cap. ${ }^{30}$

After complete fracture, we brought the two tips of the two separated SWCNTs into contact. Figures 1(d)-1(f) show a time-sequential series of the high-resolution images of the contact process. Illustrations in Figs. 1(j)-1(1) correspond to the images in Figs. 1(d)-1(f). When the tip of the SWCNT on the left-hand side approaches the opposite tip along the direction indicated by the bold arrow in Fig. 1(d) at an applied voltage of $2.2 \mathrm{~V}$, the tips contact spontaneously as shown in Fig. 1(e). The image of the SWCNT on the lefthand side in Fig. 1(e) is blurred due to vibrations. The vibrations are caused by electric charging and successive charge transfer, since the vibrations were not observed when the bias voltage was not applied. In Fig. 1(e), the current and conductance were $21 \mu \mathrm{A}$ and $0.12 G_{0}$, respectively, where $G_{0}=2 e^{2} / h$ is a conductance quantum ( $e$ is the electron charge, $h$ is the Plank constant). This result shows that the SWCNT possesses a ballistic transport property as reported previously. ${ }^{5}$ When the tip of the SWCNT on the left-hand side is compressed in the direction of the bold arrow in Fig. $1(\mathrm{e})$, a stable tubular structure is reproduced, as observed in Fig. 1(f); the two SWCNTs are joined into one. The diameter in the junction in Fig. 1(f) is slightly smaller than the minimum diameter of the SWCNT in Fig. 1(a). This observation indicates that the structure of the junction is determined not by the diameter of the SWCNT but by the structure of the cap of the tip. Comparing the mechanical properties of the reproduced SWCNT with the tube of Fig. 1(a), significant differences were hardly observed. Thus, it was found that one tubular SWCNT was produced by the contact of the two capped tips of the SWCNTs. The joining of two crossing SWCNTs was observed under the affect of defect formation by electron beam irradiation with an accelerating voltage of $1250 \mathrm{kV} .{ }^{31,32}$ In this study, the accelerating voltage was $200 \mathrm{kV}$. It is well known that defects such as vacancies and interstitials are induced into SWCNTs under electron beam irradiation at an accelerating voltage of $200 \mathrm{kV} .{ }^{33}$ However, joining was not caused by contact when force is less than $5 \mathrm{nN}$, or the bias voltage is less than $2.2 \mathrm{~V}$. This result shows that in addition to the defect formation, the combination of both applications of the force and the bias voltage of is needed for the joining to increase the reactivity of the atomic bonding of the pentagon and hexagon in carbon networks of the caps.

Figure 2 shows the variations in tensile force, minimum cross-sectional area, tensile stress and strain as a function of time, and the stress-strain relation in the tensile deformation process of Fig. 1. The times indicated by a-c in Fig. 2 cor- 

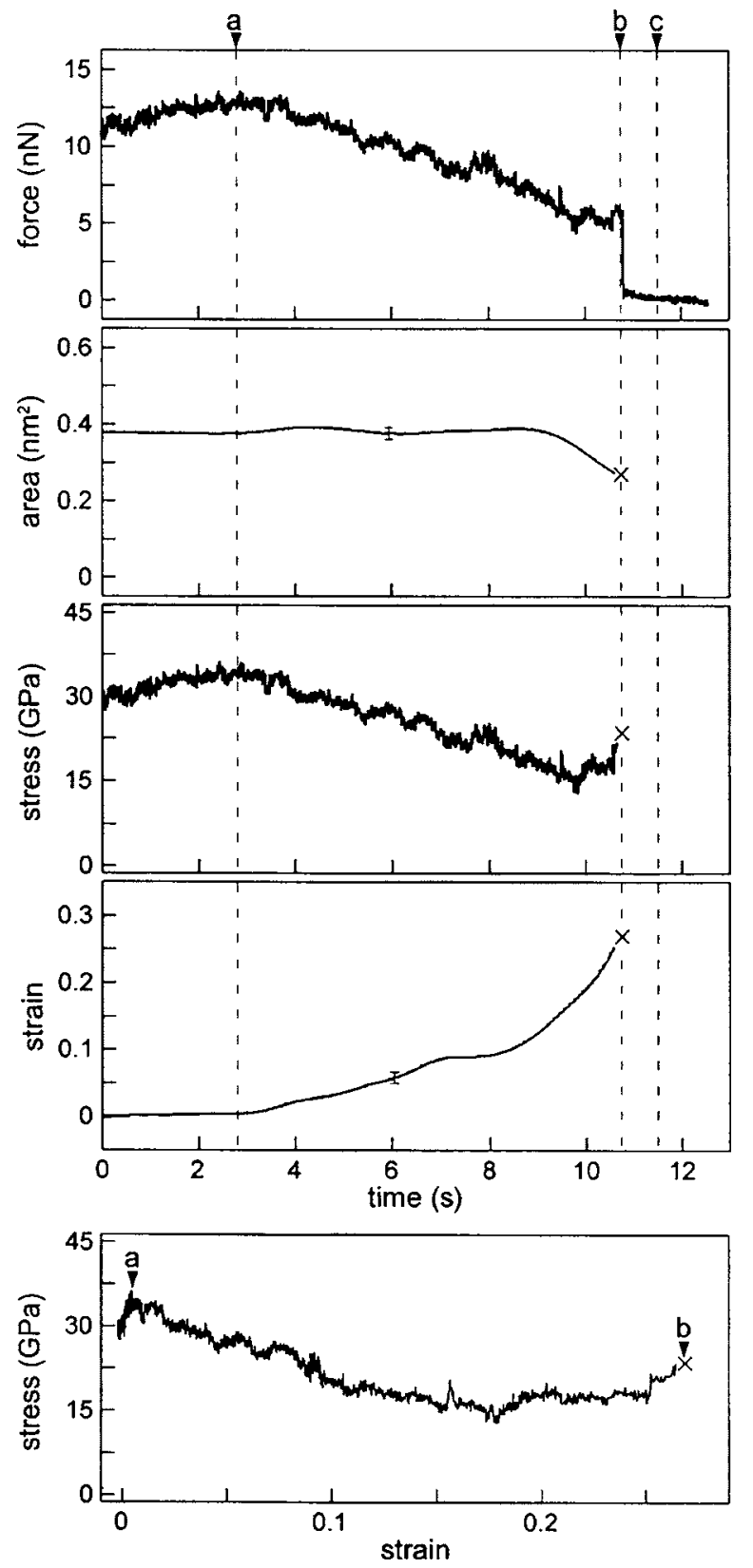

FIG. 2. Variations in tensile force, minimum cross-sectional area, tensile stress, and strain as a function of time, and the stressstrain relation during the tensile deformation process of Fig. 1. Times of a-c correspond to those at which Figs. 1(a)-1(c) were observed.

respond to those at which the high-resolution images in Figs. 1(a)-1(c) were observed. The tensile stress is calculated by $F / S$, where $F$ is the tensile force and $S$ is the minimum cross-sectional area of the SWCNT. $S$ is estimated according to $\pi\left\{(d / 2+r)^{2}-(d / 2-r)^{2}\right\}$, as used in previous reports; ${ }^{23,24} d$ is a diameter of the SWCNT, which was directly measured from the high-resolution images; and $r$ is an atomic radius of carbon, $0.077 \mathrm{~nm}$. In Fig. 2, strain increases slightly up to the time indicated by a $(2.8 \mathrm{~s})$ and both tensile stress and force increase. In this stress-strain region, a carbon hexagon network in the whole of the SWCNT extends elastically along the tensile direction. On the other hand, strain rises

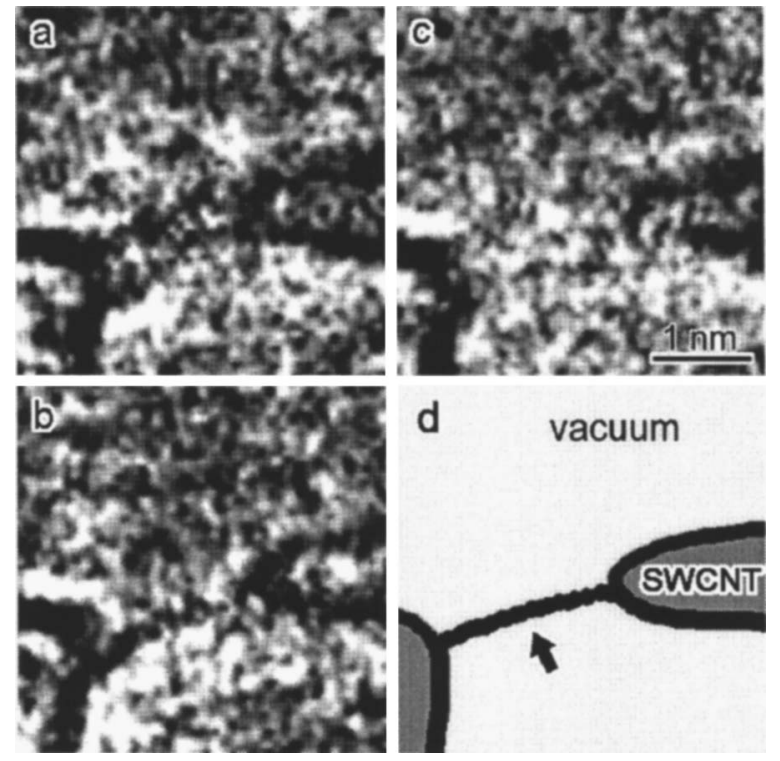

FIG. 3. (a)-(c) Time-sequential series of high-resolution images of the fracture process of a carbon wire of monatomic width and (d) an illustration of (b). A carbon monatomic wire appears between the SWCNTs before the fracture, as indicated by an arrow in (d).

rapidly from $2.8 \mathrm{~s}$, then the stress starts to decrease from 36 to $15 \mathrm{GPa}$. As previously mentioned, the point defects are introduced in the SWCNT by electron beam irradiation. The defect formation is also accelerated with mechanical deformation. It is thus considered that the decrease in stress is caused by the defect creation. The area $S$ is constant up to $9 \mathrm{~s}$ and then decreases abruptly due to buckling, as shown in Fig. 1(b). Finally, the SWCNT fractures at the time indicated by the cross in Fig. 2. From the gradient at the elastic stage in the stress-strain relation, we estimated the value of Young's modulus of the SWCNT to be $950 \pm 130 \mathrm{GPa}$. It was also determined from Fig. 2 that the tensile stress and strain at the fracture of the SWCNT were $25 \pm 1 \mathrm{GPa}$ and $0.30 \pm 0.03$, respectively. In previous reports, Young's moduli of SWCNTs have been estimated from their free-standing vibration amplitude ${ }^{18,20,21}$ or by using ropes of SWCNTs. ${ }^{24}$ In this study, the mechanical parameters relating to the tensile deformation for the isolated SWCNTs were directly measured with the observation of the atomistic structural dynamics. The value of Young's modulus in this study is in good agreement with that found in previous experiments. ${ }^{18,20,21,24}$

Next, we investigated the details of the deformation behavior during fracture. Figures 3(a)-3(c) show a timesequential series of the high-resolution images of a fracture process of a SWCNT. Figure 3(d) shows an illustration for Fig. 3(b). Two dark lines are observed between the two tips in Fig. 3(a). In Fig. 3(b), a dark line with a width of approximately $0.2 \mathrm{~nm}$ is observed between the SWCNTs, as illustrated by the arrow in Fig. 3(d). The width corresponds to a monatomic scale. Figure 3(c) shows a high-resolution image after the dark line has completely disappeared. In Fig. 4(a), the variation in tensile force measured at the deformation of Fig. 3 is shown as a function of time. Times indicated by a-c in Fig. 4(a) correspond to those in the images of Figs. $3(\mathrm{a})-3(\mathrm{c})$. The values of the tensile force decrease stepwise 

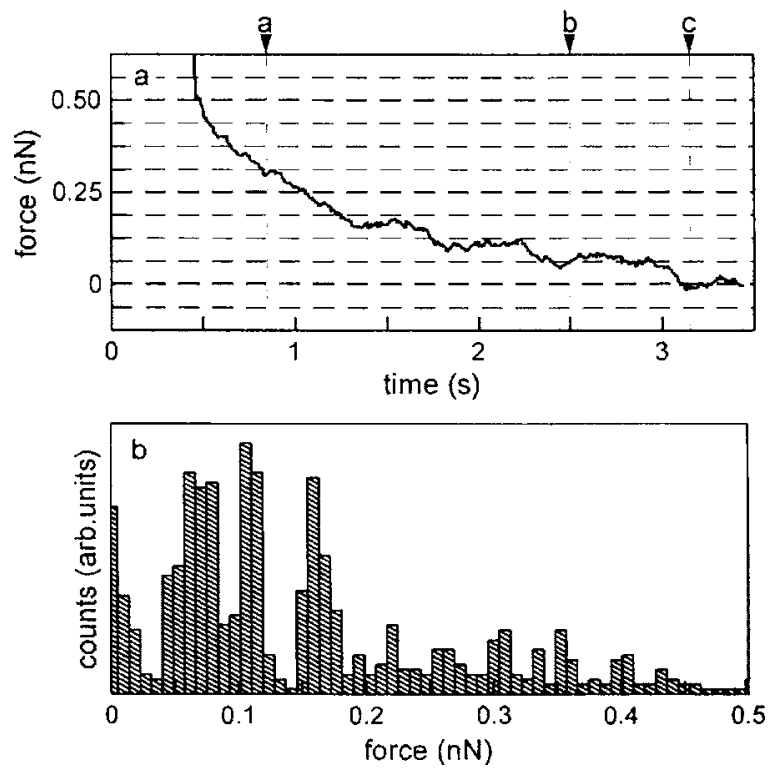

FIG. 4. (a) Variation in tensile force as a function of time and (b) histogram for the value of the tensile force corresponding to Figs. 3(a)-3(c). In the histogram, strong peaks are recognized at 0.06 , 0.11 , and $0.16 \mathrm{nN}$. The minimum value of the tensile force corresponds to the value at the smallest width of the carbon monatomic wire in Fig. 3(b).

with decreasing width of the dark line, showing that single carbon sheet breaks discretely. Figure 4(b) shows a histogram for the values of the tensile force of Fig. 4(a). Strong peaks correspond to $0.06,0.11$, and $0.16 \mathrm{nN}$. The deviation is $0.05 \mathrm{nN}$. The minimum value of the tensile force, $0.06 \mathrm{nN}$, is associated with that of the structure of the dark line with the monatomic width in Fig. 3(b). When stretched at $0.06 \mathrm{nN}$, this one-dimensional structure was elongated by approximately $0.1 \mathrm{~nm}$ before complete fracture. Troiani et al. estimated the tensile force of the monatomic wire to be $\sim 7 \mathrm{nN}$ using the tight-binding calculations. ${ }^{28}$ This calculated value is approximately $10^{2}$ times larger than the measured value in this study. The calculated static force seems to show the ideal maximal force. On the other hand, the measured force in this experiment is reduced from the maximal force by several factors, e.g. the vibration of the tips of the cantilever, the tensile deformation rate and the electron beam irradiation. The steps with a deviation of $0.05 \mathrm{nN}$ observed in Fig. 4(a) imply that the number of bonds is reduced at every unit by the same number of atoms; a part of a junction in the single monatomic sheet breaks discretely, thereby the tensile force decreases stepwise. As demonstrated in the simulations by Yakobson et al., ${ }^{34}$ a carbon monatomic wire formed in the final stage of fracture during the tensile deformation of SWCNTs. It is, therefore, inferred that a carbon monatomic wire formed in the plateau at the minimum tensile force, $0.06 \mathrm{nN}$, in Fig. 4(a).

\section{CONCLUSION}

The atomistic structural dynamics and the mechanical properties of the individual SWCNTs during tensile deformation were investigated using combined microscopy. SWCNTs were synthesized inside the combined microscope, and it was found that under stress the SWCNTs formed a neck, and subsequently fractured completely. Based on the atomistic mechanics of materials, Young's modulus of the SWCNT was estimated to be $950 \pm 130 \mathrm{GPa}$. The value of Young's modulus in this study is consistent with that found in previous reports. Before the fracture of the SWCNTs, the formation process of a carbon monatomic wire was also observed. It was shown that the tensile force of carbon wires decreased stepwise with decreasing width.

\section{ACKNOWLEDGMENTS}

We would like to thank Dr. Jose A. Torres for the helpful discussions. This work was partly supported by funds for the Special Research Project on Nanoscience and the University Research Projects of the University of Tsukuba, and by a Grant-in-Aid for Scientific Research from the Ministry of Education, Science, Sports, and Culture of Japan. Additional financial support for the present study was provided by the Nippon Sheet Glass Foundation.
${ }^{1}$ S. Iijima, Nature (London) 354, 56 (1991).

${ }^{2}$ M. R. Falvo, G. J. Clary, R. M. Taylor II, V. Chi, F. P. Brooks Jr, S. Washburn, and R. Superfine, Nature (London) 389, 582 (1997).

${ }^{3}$ T. W. Tombler, C. Zhou, L. Alexseyev, J. Kong, H. Dai, L. Liu, C. S. Jayanthi, M. Tang, and S.-Y. Wu, Nature (London) 405, 769 (2000).

${ }^{4}$ T. Kuzumaki, H. Sawada, H. Ichinose, Y. Horiike, and T. Kizuka, Appl. Phys. Lett. 79, 4580 (2001); T. Kizuka, Phys. Rev. B 59, 4646 (1999).

${ }^{5}$ S. Frank, P. Poncharal, Z. L. Wang, and W. A. de Heer, Science 280, 1744 (1998).

${ }^{6}$ J. W. G. Wildöer, L. C. Venema, A. G. Rinzler, R. E. Smalley, and C. Dekker, Nature (London) 391, 59 (1998).
${ }^{7}$ T. W. Odom, J.-L. Huang, P. Kim, and C. M. Lieber, Nature (London) 391, 62 (1998).

${ }^{8}$ S. Sanvito, Y.-K. Kwon, D. Tománek, and C. J. Lambert, Phys. Rev. Lett. 84, 1974 (2000).

${ }^{9}$ C. Q. Sun, H. L. Bai, B. K. Tay, S. Li, and E. Y. Jiang, J. Phys. Chem. B 107, R7544 (2003).

${ }^{10}$ D. H.Robertson, D. W.Brenner, and J. W. Mintmire, Phys. Rev. B 45, R12592 (1992).

${ }^{11}$ B. I. Yakobson, C. J. Brabec, and J. Bernholc, Phys. Rev. Lett. 76, 2511 (1996).

${ }^{12}$ J. P. Lu, Phys. Rev. Lett. 79, 1297 (1997).

${ }^{13}$ C. F. Cornwell and L. T. Wille, Solid State Commun. 101, 555 (1997).

${ }^{14}$ E. Hernández, C. Goze, P. Bernier, and A. Rubio, Phys. Rev. Lett. 
80, 4502 (1998).

${ }^{15}$ D. Sánchez-Portal, E. Artacho, J. M. Soler, A. Rubio, and P. Ordejón, Phys. Rev. B 59, 12678 (1999).

${ }^{16}$ V. N. Popov, V. E. Van Doren, and M. Balkanski, Phys. Rev. B 61, 3078 (2000).

${ }^{17}$ Y. Jin and F. G. Yuan, Compos. Sci. Technol. 63, 1507 (2003).

${ }^{18}$ M. M. J. Treacy, T. W. Ebbesen, and J. M. Gibson, Nature (London) 381, 678 (1996).

${ }^{19}$ E. W. Wong, P. E. Sheehan, and C. M. Lieber, Science 277, 1971 (1997).

${ }^{20}$ A. Krishnan, E. Dujardin, T. W. Ebbesen, P. N. Yianilos, and M. M. J. Treacy, Phys. Rev. B 58, 14013 (1998).

${ }^{21}$ P. Poncharal, Z. L. Wang, D. Ugarte, and W. A. de Heer, Science 283, 1513 (1999).

${ }^{22}$ J.-P. Salvetat, G. A. D. Briggs, J.-M. Bonard, R. R. Bacsa, A. J. Kulik, T. Stöckli, N. A. Burnham, and L. Forró, Phys. Rev. Lett. 82, 944 (1999).

${ }^{23}$ M.-F. Yu, O. Lourie, M. J. Dyer, K. Moloni, T. F. Kelly, and R. S. Ruoff, Science 287, 637 (2000)

${ }^{24}$ M.-F. Yu, B. S. Files, S. Arepalli, and R. S. Ruoff, Phys. Rev. Lett. 84, 5552 (2000).
${ }^{25}$ S. Akita, H. Nishijima, T. Kishida, and Y. Nakayama, Jpn. J. Appl. Phys., Part 1 39, 3724 (2000).

${ }^{26}$ T. Kizuka, H. Ohmi, T. Sumi, K. Kumazawa, S. Deguchi, M. Naruse, S. Fujisawa, S. Sasaki, A. Yabe, and Y. Enomoto, Jpn. J. Appl. Phys., Part 1 40, L170 (2001).

${ }^{27}$ J. Yamashita, H. Hirayama, Y. Ohshima, and K. Takayanagi, Appl. Phys. Lett. 74, 2450 (1999).

${ }^{28}$ H. E. Troiani, M. Miki-Yoshida, G. A. Camacho-Bragado, M. A. L. Marques, A. Rubio, J. A. Ascencio, and M. Jose-Yacaman, Nano Lett. 3, 751 (2003).

${ }^{29}$ S. Iijima and T. Ichihashi, Nature (London) 363, 603 (1993).

${ }^{30}$ S. Iijima, M. Yudasaka, R. Yamada, S. Bandow, K. Suenaga, F. Kokai, and K. Takahashi, Chem. Phys. Lett. 309, 165 (1999).

${ }^{31}$ M. Terrones, F. Banhart, N. Grobert, J.-C. Charlier, H. Terrones, and P. M. Ajayan, Phys. Rev. Lett. 89, 075505 (2002).

${ }^{32}$ M. Terrones, H. Terrones, F. Banhart, J.-C. Charlier, and P. M. Ajayan, Science 288, 1226 (2000).

${ }^{33}$ F. Banhart, Rep. Prog. Phys. 62, 1181 (1999).

${ }^{34}$ B. I. Yakobson, M. P. Campbell, C. J. Brabec, and J. Bernholc, Comput. Mater. Sci. 8, 341 (1997). 